KYUNGPOOK Math. J. 54(2014), 595-606

http://dx.doi.org/10.5666/KMJ.2014.54.4.595

\title{
Special Right Jacobson Radicals for Right Near-rings
}

RAVi SRINIVASA RAO*

Department of Mathematics, R. V.R. \& J. C. College of Engineering, Chandramoulipuram, Chowdavaram, Guntur-522019, Andhra Pradesh, India

e-mail : dr_rsrao@yahoo.com

Korrapati Siva PRASAD

Department of Mathematics, Acharya Nagarjuna University, Nagarjuna Nagar522510, Guntur (Dist.), Andhra Pradesh, India

e-mail : siva235prasad@yahoo.co.in

ABSTRACT. In this paper three more right Jacobson-type radicals, $J_{g_{\nu}}^{r}$, are introduced for near-rings which generalize the Jacobson radical of rings, $\nu \in\{0,1,2\}$. It is proved that $J_{g_{\nu}}^{r}$ is a special radical in the class of all near-rings. Unlike the known right Jacobson semisimple near-rings, a $J_{g_{\nu}}^{r}$-semisimple near-ring $R$ with DCC on right ideals is a direct sum of minimal right ideals which are right $R$-groups of type- $g_{\nu}, \nu \in\{0,1,2\}$. Moreover, a finite right $g_{2}$-primitive near-ring $R$ with $e R e$ a non-ring is a near-ring of matrices over a near-field (which is isomorphic to $e R e$ ), where $e$ is a right $g_{2}$-primitive idempotent in $R$.

\section{Introduction}

Special radicals for near-rings are introduced in [1] by G. L. Booth and N. J. Groenewald using equiprime near-rings. Among the known left Jacobson-type radicals, $J_{3}, J_{3(0)}$ are the only special radicals in the class of zero-symmetric near-rings and in the class of all near-rings respectively.

Srinivasa Rao and Siva Prasad $[6,7]$ introduced and studied $J_{\nu}^{r}$, the right Jacobson radical type- $\nu, \nu \in\{0,1,2\}$. In $[9,10]$ Srinivasa Rao and Siva Prasad along with T. Srinivas showed that $J_{\nu}^{r}$ is a Kurosh-Amitsur radical in the Fuchs variety $\mathcal{F}$ of all near-rings $R$ in which the constant part $R_{c}$ of $R$ is an ideal of $R, \nu \in\{0,1,2\}$. But $J_{\nu}^{r}$ is not s-hereditary in the class of all zero-symmetric near-rings and hence it is not an ideal-hereditary radical in that class, $\nu \in\{0,1,2\}$.

Also in [5]([11]) Srinivasa Rao and Siva Prasad (along with T. Srinivas) intro-

* Corresponding Author.

Received December 26, 2011; accepted December 18, 2012.

2010 AMS Mathematics Subject Classification: 16 Y30.

This paper was presented at the 11th International Conference on the 'Theory of Radicals, Rings and Modules' held at the Sultan Qaboos University, Muscat, Oman, during 20-26 January, 2012. 
duced and studied the right Jacobson type of radical $J_{\nu(e)}^{r}, \nu \in\{1,2\}\left(J_{0(e)}^{r}\right)$ and showed that it is a Kurosh-Amitsur radical in the class of all near-rings and is an ideal hereditary Kurosh-Amitsur radical in the class of all zero-symmetric nearrings. Moreover, they are special radicals in the class of all near-rings.

In this paper we introduce three more right Jacobson radicals, $J_{g_{\nu}}^{r}, \nu \in\{0,1,2\}$. We show that they are special radicals in the class of all near-rings. So, in the class of all near-rings, they are Kurosh-Amitsur radicals, their semisimple classes are hereditary and radicals classes are c-hereditary. Unlike the known right Jacobson semisimple near-rings, a $J_{g_{\nu}}^{r}$-semisimple near-ring $R$ with DCC on right ideals is a direct sum of right ideals which are right $R$-groups of type- $g_{\nu}, \nu \in\{0,1.2\}$. A finite right $g_{2}$-primitive near-ring $R$ with $e R e$ a non-ring is a near-ring of matrices over a near-field (which is isomorphic to $e R e$ ), where $e$ is a right $g_{2}$-primitive idempotent in $R$.

Near-rings considered are right near-rings (not necessarily zero-symmetric) and $R$ is a near-ring. Now we present some definitions and results of [6] and [7].

A group $(G,+)$ is called a right $R$-group if there is a mapping $((g, r) \rightarrow g r)$ of $G \times R$ into $G$ such that $(1)(g+h) r=g r+h r,(2) g(r s)=(g r) s$ for all $g, h \in G$ and $r, s \in R$. A subgroup (normal subgroup) $H$ of a right $R$-group $G$ is called an $R$-subgroup (ideal) of $G$ if $h r \in H$ for all $h \in H$ and $r \in R$.

Let $G$ be a right $R$-group. An element $g \in G$ is called a generator of $G$ if $g R=G$ and $g(r+s)=g r+g s$ for all $r, s \in R$. $G$ is said to be monogenic if $G$ has a generator. $G$ is said to be simple if $G \neq\{0\}$ and $G$, and $\{0\}$ are the only ideals of $G$.

A monogenic right $R$-group $G$ is said to be a right $R$-group of type-0 if $G$ is simple.

A right $R$-group $G$ of type-0 is said to be of type- 1 if $G$ has exactly two $R$ subgroups, namely $\{0\}$ and $G$.

A right $R$-group $G$ of type-0 is said to be of type-2 if $g R=G$ for all $0 \neq g \in G$.

Note that a right $R$-group of type- 2 is of type- 1 and a right $R$-group of type- 1 is of type- 0 .

Let $\nu \in\{0,1,2\}$. A right modular right ideal $K$ of $R$ is called right $\nu$-modular if $R / K$ is a right $R$-group of type- $\nu$.

An ideal $P$ of $R$ is called right $\nu$-primitive if $P$ is the largest ideal of $R$ contained in a right $\nu$-modular right ideal of $R . R$ is called a right $\nu$-primitive near-ring if $\{0\}$ is a right $\nu$-primitive ideal of $R$.

Now we present some definitions of [11] and [5].

Let $G$ be a right $R$-group of type- $\nu, \nu \in\{0,1,2\}$. Suppose that $G 0=\{0\}$ for $\nu=0$ and $P$ is the largest ideal of $R$ contained in $(0: G)=\{r \in R \mid G r=\{0\}\}$. Then $G$ is said to be a right $R$-group of type- $\nu(e)$ if $0 \neq g \in G, r_{1}, r_{2} \in R$ and $g x r_{1}=g x r_{2}$ for all $x \in R$ implies $r_{1}-r_{2} \in P$.

A right modular right ideal $K$ of $R$ is called right $\nu(e)$-modular if $R / K$ is a right $R$-group of type- $\nu(e)$.

Let $G$ be a right $R$-group of type- $\nu(e)$. Then $(0: \mathrm{G})$ is an ideal of $R$ and is called a right $\nu(e)$-primitive ideal of $R$. 
A near-ring $R$ is called right $\nu(e)$-primitive if $\{0\}$ is a right $\nu(e)$-primitive ideal of $R$.

A near-ring $\mathrm{R}$ is called an equiprime near-ring [2] if $0 \neq \mathrm{a} \in \mathrm{R}, \mathrm{x}, \mathrm{y} \in \mathrm{R}$ and arx $=$ ary for all $\mathrm{r} \in \mathrm{R}$, implies $\mathrm{x}=\mathrm{y}$. An ideal $\mathrm{I}$ of $\mathrm{R}$ is called equiprime if $\mathrm{R} / \mathrm{I}$ is an equiprime near-ring. Moreover, an equiprime near-ring is zero-symmetric.

It is known that a near-ring $\mathrm{R}$ is equiprime if and only if ([2])

1. $\mathrm{x}, \mathrm{y} \in \mathrm{R}$ and $\mathrm{xRy}=\{0\}$ implies $\mathrm{x}=0$ or $\mathrm{y}=0$.

2. If $\{0\} \neq \mathrm{I}$ is an invariant subnear-ring of $\mathrm{R}, \mathrm{x}, \mathrm{y} \in \mathrm{R}$ and $\mathrm{ax}=$ ay for all $\mathrm{a} \in \mathrm{I}$ implies $\mathrm{x}=\mathrm{y}$.

In [1], G. L. Booth and N. J. Groenewald defined special radicals for near-rings. A class $\mathcal{E}$ consisting of equiprime near-rings is called a special class if it is hereditary and closed under left invariant essential extensions. If $\mathcal{R}$ is the upper radical in the class of all near-rings determined by a special class of near-rings, then $\mathcal{R}$ is called a special radical. A class of near-rings $\mathcal{E}$ is said to satisfy condition $F_{l}$ if $J \triangleleft I \triangleleft R$ and $I$ is left invariant in $R$ and $I / J \in \mathcal{E}$ implies $J \triangleleft R$. We need the following theorem:

Theorem 1.1. ([12]) Let $\mathcal{E}$ be a class of zero-symmetric near-rings. If $\mathcal{E}$ is regular, closed under essential left invariant extensions and satisfies condition $\left(\mathrm{F}_{l}\right)$, then $\mathcal{R}$ $:=\mathcal{U} \mathcal{E}$ is a c-hereditary radical class in the variety of all near-rings, $\mathcal{S} \mathcal{R}=\overline{\mathcal{E}}$ and $\mathcal{S} \mathcal{R}$ is hereditary. So, $\mathcal{R}(\mathrm{R})=\cap\{\mathrm{I} \triangleleft \mathrm{R} \mid \mathrm{R} / \mathrm{I} \in \mathcal{E}\}$ for any near-ring $\mathrm{R}$.

\section{Right Jacobson Radicals of Type- $g_{\nu}$}

Let $G$ be a right $R$-group and $T$ be a subset of $G$. Then $(0: T):=\{r \in R \mid t r=0$ for all $t \in T\}$. By Proposition 3.7 of [11], if $G$ is a right $R$-group of type- 0 and $G 0=\{0\}$, then there is a largest ideal of $R$ contained in $(0: G)$. Moreover, by Proposition 3.1 of [5], if $G$ is a right $R$-group of type- $\nu$, then $G 0=\{0\}, \nu \in\{1,2\}$. Definition 2.1. Let $\nu \in\{0,1,2\}$. Let $G$ be right $R$-group of type- $\nu$ and $G 0=\{0\}$ for $\nu=0$, and $T$ be the set of all generators of the right $R$-group $G$. Then $G$ is said to be a right $R$-group of type- $g_{\nu}$ if $(0: T)=P$, where $\mathrm{P}$ is the largest ideal of $R$ contained in $(0: G)$.

We present an example of a right $R$-group of type- $g_{0}$ which is not of type- $g_{1}$.

Example 2.2. Let $(G,+)$ be a finite non-abelian simple group. Since $\{0\}$ is the maximal normal subgroup of $(G,+),\{0\}$ is the maximal right ideal of $M_{0}(G)$ and hence $M_{0}(G)$ is a right $M_{0}(G)$-group of type- 0 . This example was considered in [7] and it was shown that $M_{0}(G)$ is not a right $M_{0}(G)$-group of type-1. Each $0 \neq h \in G$ give rise to the inner automorphism $t_{h}$ of $G$ defined by $t_{h}(x)=h+x-h$ for all $x \in G$. Clearly, a generator of the right $M_{0}(G)$-group $M_{0}(G)$ is an automorphism of $(G,+)$. Let $T$ be the set of all automorphisms of $G$. Suppose that for some $t \in M_{0}(G)$ and $0 \neq h \in G, t_{h} t=0$. Now $0=\left(t_{-h}\right) t_{h} t=\left(t_{h}\right)^{-1} t_{h} t=t$. Therefore $\{0\}=\left(0: t_{h}\right)=(0: T)$. Since the largest ideal of $M_{0}(G)$ contained in $\left(0: M_{0}(G)\right)$ is $\{0\}, M_{0}(G)$ is a right $M_{0}(G)$-group of type- $g_{0}$ but not of type- $g_{1}$.

Now we present an example of a right $R$-group of type- $g_{1}$ which is not of type- $g_{2}$. 
Example 2.3. Let $(G,+)$ be a finite cyclic group of prime order $p$, where $p \neq 2$. Since $\{0\}$ is the only proper subgroup of $G,\{0\}$ is the only proper right $M_{0}(G)$ subgroup of $M_{0}(G)$. Therefore, $M_{0}(G)$ is a right $M_{0}(G)$-group of type-1. Clearly, $M_{0}(G)$ is not a right $M_{0}(G)$-group of type-2, as $M_{0}(G)$ is not a near-field. This example was considered in [7]. A a generator of the right $M_{0}(G)$-group $M_{0}(G)$ an is automorphism $(G,+)$. We know that $G$ has $p-1$ automorphisms. Let $T$ be the set of all these automorphisms. Suppose that for some $s \in M_{0}(G)$ and $t \in T, t s=0$. Now $0=\left(t^{-1}\right) t s=s$. So $\{0\}=(0: t)=(0: T)$. Since the largest ideal of $M_{0}(G)$ contained in $\left(0: M_{0}(G)\right)$ is $\{0\}, M_{0}(G)$ is a right $M_{0}(G)$-group of type- $g_{1}$ but not of type- $g_{2}$.

The following are examples of right $R$-groups of type- $g_{2}$.

Example 2.4. Let $R$ be a near-field. Then $R$ is a right $R$-group of type-2. Clearly, $R$ is also a right $R$-group of type- $g_{2}$.

Example 2.5. Let $(R,+)$ be a group and let $K$ be a subgroup of $(R,+)$ of index 2. The trivial multiplication on $(R,+)$ determined by $R \backslash K$ is given by $a . b=a$ if $b \in R \backslash K$ and 0 if $b \in K$. Now $(R,+,$.$) is a near-ring. It is clear that K$ is a maximal (right) ideal of $R$. Let $a \in R \backslash K$. Now $R=K \cup a+K$. It can be easily verified that $a+K$ is the generator of the right $R$-group $R / K$. So $R / K$ is a right $R$-group of type- 2 and $(0: a+K)=(0: R / K)$ is the largest ideal of $R$ contained in $(0: R / K)$. Hence $R / K$ is a right $R$-group of type- $g_{2}$.

Now we introduce some notions related to the right $R$-groups of type- $g_{\nu}$.

Definition 2.6. Let $\nu \in\{0,1,2\}$ and $K$ be a right modular right ideal of $R$. Then $K$ is said to be right $g_{\nu}$-modular right ideal of $R$ if $R / K$ is a right $R$-group of type- $g_{\nu}$.

Definition 2.7. Let $\nu \in\{0,1,2\}$. An ideal $P$ of $R$ is called a right $g_{\nu}$-primitive ideal of $R$ if $P$ is the largest ideal of $R$ contained in $(0: G):=\{r \in R \mid G r=\{0\}\}$ for some right $R$-group $G$ of type- $g_{\nu}$.

Definition 2.8. Let $\nu \in\{0,1,2\}$. A near-ring $R$ is called a right $g_{\nu \text {-primitive }}$ near-ring if $\{0\}$ is a right $g_{\nu}$-primitive ideal of $R$.

Definition 2.9. Let $\nu \in\{0,1,2\}$. The intersection of all right $g_{\nu}$-primitive ideals of $R$ is called the right Jacobson radical of $R$ of type- $g_{\nu}$ and is denoted by $J_{g_{\nu}}^{r}(R)$. If $R$ has no right $g_{\nu}$-primitive ideals, then $J_{g_{\nu}}^{r}(R)$ is defined to be $R$. of $R$.

Note that if $R$ is a ring then $J_{g_{\nu}}^{r}(R)=J(R)$, where $J$ is the Jacobson radical

By Proposition 3.1 of [11], for a right $R$-group $G, G 0=\{0\}$ if and only if $G R_{c}=\{0\}$. Since for a right $R$-group $G$ of type- $g_{\nu}, G 0=\{0\}, R_{c}$ is contained in $(0: g)$ for every generator $g$ of $G$. So $R_{c} \subseteq P$ for every right $g_{\nu}$-primitive ideal $P$ of $R$. Hence a right $g_{\nu}$-primitive ideal $P$ of $R$ is invariant. This shows that a right $g_{\nu}$-primitive near-ring is zero-symmetric. 
Proposition 2.10. Let $\nu \in\{0,1,2\}$. An ideal $P$ of $R$ is a right $g_{\nu}$-primitive ideal of $R$ if and only if $P$ is the largest ideal of $R$ contained in a right $g_{\nu}$-modular right ideal of $R$.

Proof. Let $P$ be a right $g_{\nu}$-primitive ideal of $R$. There is a right $R$-group $G$ of type$g_{\nu}$ such that $P$ is the largest ideal of $R$ contained in $(0: G)$. Let $g_{0}$ be a generator of the right $R$-group $G$. The mapping $r \rightarrow g_{0} r$ is a right $R$-homomorphism of $R$ on to $G$ with kennel $K:=\left(0: g_{0}\right)$. So $R / K$ is right $R$-isomorphic to $G$ (as right $R$-groups). Now $K$ is a right $g_{\nu}$-modular right ideal of $R$ and $P$ is contained in $K$. Let $Q$ is the largest ideal of $R$ contained in $K$. Now $G Q=\{0\}$, that is, $Q \subseteq(0: G)$ as $R Q \subseteq Q$, $\mathrm{Q}$ being invariant ideal of $R$. Since $P$ is the largest ideal of $R$ contained in $(0: G)$, $Q \subseteq P$. Now $P \subseteq Q$ as $Q$ is the largest ideal of $R$ contained in $K$. Therefore $P=Q$, that is, $P$ is the largest ideal of $R$ contained in $K$. On the other hand suppose that $P$ is the largest ideal of $R$ contained in a right $g_{\nu}$-modular right ideal $K$ of $R$. Now $G:=R / K$ is a right R-group of type- $g_{\nu}$. We have $(0: G)=(0: R / K)=(K: R)$ and $R P \subseteq P$ as $\mathrm{P}$ is an invariant ideal of $R$. So $P \subseteq(K: R)$. Let $T$ be the largest ideal of $R$ contained in $(K: R)=\{r \in R \mid R r \subseteq K\}$. Since $P$ is an invariant ideal of $R$, and $P \subseteq T, \mathrm{~T}$ is an invariant ideal of $R$. So $R T \subseteq T$. Let $K$ be right modular by $e$. Now $r-e r \in K$ for all $r \in R$. We have $t-e t \in K$ for all $t \in T$. Since $R T \subseteq T, T \subseteq K$. Since $P$ is the largest ideal of $R$ contained in $K, T \subseteq P$. So $T=P$. Now $P$ is the largest ideal of $R$ contained in $(K: R)$ and hence $P$ is a right $g_{\nu}$-primitive ideal of $R$.

Proposition 2.11. Let $\nu \in\{0,1,2\}$. An ideal $P$ of $R$ is a right $g_{\nu}$-primitive ideal of $R$ if and only if $R / P$ is a right $g_{\nu}$-primitive near-ring.

Proof. Let $\nu \in\{0,1,2\}$ and $P$ be an ideal of $R$. Suppose that $P$ is a right $g_{\nu^{-}}$ primitive ideal of $R$. So, we get a right $g_{\nu^{-}}$modular right ideal $M$ of $R$ such that $P$ is the largest ideal of $R$ contained in $M$. Now $M / P$ is a right $g_{\nu}$-modular right ideal of $R / P$. Since $P$ is the largest ideal of $R$ contained in $M$, the zero ideal of $R / P$ is the largest ideal of $R / P$ contained in $M / P$. Therefore, $R / P$ is a right $g_{\nu}$-primitive near-ring. Suppose now that $R / P$ is a right $g_{\nu}$-primitive near-ring. So, we get a right $g_{\nu}$-modular right ideal $M / P$ of $R / P$ such that the zero ideal of $R / P$ is the largest ideal of $R / P$ contained in $M / P$. Clearly, $M$ is a right $g_{\nu}$-modular right ideal of $R$. Since the zero ideal of $R / P$ is the largest ideal of $R / P$ contained in $M / P, P$ is the largest ideal of $R$ contained in $M$. Therefore, $P$ is a right $g_{\nu}$-primitive ideal of $R$.

Proposition 2.12. $J_{g_{\nu}}^{r}$ is the Hoehnke radical determined by the class of all right $g_{\nu}$-primitive near-rings, $\nu \in\{0,1,2\}$.

Theorem 2.13. Let $G$ be a right $R$-group of type- $g_{\nu}$ and $S$ be an invariant subnearring (and right ideal for $\nu=0$ ) of $R$ with $G S \neq\{0\}$. Then $G$ is a right $S$-group of type-g $g_{\nu}, \nu \in\{0,1,2\}$. 
Proof. If $G$ is a right $R$-group of type-0 and $S$ is an invariant subnear-ring and right ideal of $R$ with $G S \neq\{0\}$, then under the restriction of $G$ to $S$, by Theorem 3.2 of [9], $G$ is a right $S$-group type-0. Also if $G$ be a right $R$-group of type- $\nu$ and $S$ is an invariant subnear-ring of $R$ with $G S \neq\{0\}$, then under the restriction of $G$ to $S$, by Theorems 3.1 and 3.2 of [10], $G$ is a right $S$-group type- $\nu$, where $\nu \in\{1,2\}$. Therefore $G$ is a right $S$-group of type- $\nu, \nu \in\{0,1,2\}$. Let $A$ be the set of generators of the right $R$-group $G$ and $P$ be the largest ideal of $R$ contained in $(0: G)_{R}:=\{r \in R \mid G r=\{0\}\}$. A generator of the right $R$-group $G$ is also a generator of the right $S$-group $G$. From the proof of Theorem 3.10 of [9] (and Theorems 3.9 and 3.10 of [10] for $\nu \in\{1,2\}$ ) as the extension of $G$ from $S$ to $R$ coincides with the action of $G$ on $R$, it follows that a generator of the right $S$-group $G$ is also a generator of the right $R$-group $G$. So $A$ is the set of generators of the right $S$-group $G$. We have $P=(0: A)=\{r \in R \mid$ ar $=0$ for all $a \in A\}$. Now $P \cap S=(0: A) \cap S=\{s \in S \mid A s=\{0\}\}$. Let $Q$ be the largest ideal of $S$ contained in $(0: G)_{S}:=\{s \in S \mid G s=\{0\}\}=(0: G) \cap S$. Clearly $P \cap S \subseteq(0: G)_{S}$. By the definition of $Q, P \cap S \subseteq Q$. Since $A Q=\{0\}, Q \subseteq P$. So $Q \subseteq P \cap S$. Therefore $Q=P \cap S$. Hence $G$ is a right $S$-group of type- $g_{\nu}$.

Proposition 2.14. A right $R$-group of type- $g_{\nu}$ is an $R$-group of type- $\nu(e), \nu \in$ $\{0,1,2\}$.

Proof. Let $G$ be a right $R$-group of type- $g_{\nu}, \nu \in\{0,1,2\}$. So $G$ is a right $R$-group of type- $\nu$. In view of Remark 3.9 of [11] $G$ is a right $R$-group of type- $\nu(e)$ if $r, s \in R$ and $g r=g s$ for all $g \in G$, then $r-s \in P$ where $P$ is the largest ideal of $R$ contained in $(0: G):=\{r \in R \mid G r=\{0\}\}$. Let $g r=g s$ for all $g \in G, r, s, \in R$ and $P$ be the largest ideal of $R$ contained in $(0: G)$. Let $A$ be the set of all generators of the right $R$-group $G$. Now $a r=a s$ for all $a \in A$. Since each $a \in A$ is distributive, $a(r-s)=0$ for all $a \in A$. Therefore $r-s \in P$ as $P=(0: A)$. Hence $G$ is a right $R$-group of type- $\nu(e)$.

Remark 2.15. If $G$ is a right $R$-group of type- $\nu(e)$, then by Proposition 3.12 of [11], $(0: G):=\{r \in R \mid G r=\{0\}\}$ is an ideal of $R$. Also, by Theorem 3.24 of [11], a right $g_{\nu}$-primitive near-ring is an equiprime near-ring.

Definition 2.16. Let $G$ be a right $R$-group of type- $g_{\nu}, \nu \in\{0,1,2\}$. Then $G$ is called faithful if $(0: G)=\{0\}$.

Theorem 2.17. Let $G$ be a faithful right $S$-group of type- $g_{\nu}$ and $S$ be an essential left invariant ideal of $R$. Then $G$ is a faithful right $R$-group of type- $g_{\nu}, \nu \in\{0,1,2\}$.

Proof. Let $h_{0}$ be a generator of the right $S$-group $G$. From the proof of Theorem 3.10 of [9], for $h \in H, r \in R$ the operation defined by $h r:=h_{0}(s r)$ if $h=h_{0} s, s \in S$, makes $G$ a right $R$-group and is an extension the action of $G$ on $S$ to $R$. Moreover, Theorem 3.10 of [9] and Theorems 3.9 and 3.10 of [10], $G$ is a right $R$-group of type- $\nu$, for $\nu \in\{1,2\}$. Since $G$ is a right $R$-group of type- $\nu(e)$, by Theorem 3. 33 of [11] and Theorem of [5], $G$ is a faithful $R$-group of type- $\nu(e)$. Let $A$ be the set of 
all generators of the right $S$-group $G$. Now $(0: G)_{S}:=\{s \in S \mid G s=\{0\}\}=\{0\}$. We have $\{0\}=(0: A)_{S}:=\{s \in S \mid A s=\{0\}\}$. Since $G$ is a faithful right $R$-group, $(0: G)_{R}:=\{r \in R \mid G r=\{0\}\}=\{0\}$. From the proof of Theorem 3.10 of [9], it can be easily seen that a generator of the right $S$-group $G$ is also a generator of the right $R$-group $G$. So $A$ is the set of generators of the right $R$-group $G$. Suppose that $r \in(0: A)$. Now $A r=\{0\}$. So $\{0\}=(A r) S=A(r S)$ and hence $r S=\{0\}$ as $r S \subseteq S$. Since $S$ is an ideal, $K S=\{0\}$ and $S$ is a prime near-ring, we have $K=\{0\}$, where $K$ is the ideal of $R$ generated by $r$. Therefore $r=0$ and hence $(0: A)_{R}=\{0\}$. So $G$ is a faithful right $R$-group of type- $g_{\nu}$.

From the above theorem we have:

Theorem 2.18. The class of all right $g_{\nu}$-primitive near-rings is closed under essential left invariant extensions, $\nu \in\{0,1,2\}$.

In view of Theorem 1.1, we have the following:

Theorem 2.19. Let $\nu \in\{0,1,2\}$. Let $\mathcal{E}$ be the class of all right $g_{\nu}$-primitive near-rings and $\mathcal{U} \mathcal{E}$ be the upper radical class determined by $\mathcal{E}$. Then $\mathcal{U} \mathcal{E}$ is a $c$ hereditary Kurosh-Amitsur radical class in the variety of all near-rings with hereditary semisimple class $\mathcal{S} \mathcal{E}=\mathcal{E}$. So, $J_{g_{\nu}}^{r}$ is a Kurosh-Amitsur radical in the class of all near-rings and for any ideal $I$ of $R, J_{g_{\nu}}^{r}(I) \subseteq J_{g_{\nu}}^{r}(R) \cap I$ with equality, if $I$ is left invariant.

Theorem 2.20. $J_{g_{\nu}}^{r}$ is an ideal-hereditary Kurosh-Amitsur radical in the class of all zero-symmetric near-rings.

Theorem 2.21. $J_{g_{\nu}}^{r}$ is a special radical in the class of all near-rings.

\section{Examples}

In this section we present some examples of near-rings $R$ and their right $R$ groups to show that the present right Jacobson radicals are distinct from the known right Jacobson radicals of near-rings. Now we present an example of a right $R$-group of type- $\nu(e)$ which is not of type- $g_{\nu}, \nu \in\{0,1,2\}$.

Proposition 3.1. If $\mathrm{G}$ be a finite group and $\mathrm{G}$ has a subgroup of index two, then $\mathrm{M}_{0}(\mathrm{G})$ is a right 2(e)-primitive near-ring.

Proof. Let $\mathrm{G}$ be a finite group and $\mathrm{H}$ be a subgroup of $\mathrm{G}$ of index 2. So $\mathrm{H}$ is a normal subgroup of $\mathrm{G}$. Let $\mathrm{R}=\mathrm{M}_{0}(\mathrm{G})$. Then $\mathrm{R} / \mathrm{K}$ is a right R-group of type-2(e), where $\mathrm{K}=(\mathrm{H}: \mathrm{G})=\{\mathrm{r} \in \mathrm{R} \mid \mathrm{r}(\mathrm{g}) \in \mathrm{H}$, for all $\mathrm{g} \in \mathrm{G}\}$. To show this we consider the two distinct cosets $\mathrm{H}$ and $\mathrm{H}+\mathrm{a}$ of $\mathrm{H}$ in $\mathrm{G}$. Now $\mathrm{G}=\mathrm{H} \cup \mathrm{H}+\mathrm{a}, \mathrm{H}$ and $\mathrm{H}+$ a are disjoint sets. $\mathrm{K}$ is a right ideal of $\mathrm{R}$ which is right modular by the identity element of $R$. So $R / K$ is a monogenic right $R$-group. Now we show that $R / K$ is a right $\mathrm{R}$-group of type- 2 . Let $0 \neq \mathrm{r}+\mathrm{K} \in \mathrm{R} / \mathrm{K}$. $(\mathrm{r}+\mathrm{K}) \mathrm{R}=\mathrm{R} / \mathrm{K}$ if and only if 
there is an $\mathrm{s} \in \mathrm{R}$ such that $(\mathrm{r}+\mathrm{K}) \mathrm{s}=1+\mathrm{K}$, that is, $1-\mathrm{rs} \in \mathrm{K}$. Let $\mathrm{P}_{1}=\{\mathrm{x} \in \mathrm{G}$ $\mid \mathrm{r}(\mathrm{x}) \in \mathrm{H}\}$ and $\mathrm{P}_{2}=\{\mathrm{x} \in \mathrm{G} \mid \mathrm{r}(\mathrm{x}) \in \mathrm{H}+\mathrm{a}\}$. Let $\mathrm{b} \in \mathrm{P}_{2}$ and $\mathrm{r}(\mathrm{b})=\mathrm{h}^{\prime}+\mathrm{a}, \mathrm{h}^{\prime} \in$ H. Define $\mathrm{s}: \mathrm{G} \rightarrow \mathrm{G}$ by $\mathrm{s}(\mathrm{g})=\mathrm{b}$, if $\mathrm{g} \in \mathrm{H}+\mathrm{a}$, and 0 , if $\mathrm{g} \in \mathrm{H}$. We have $\mathrm{s} \in \mathrm{R}$. For $\mathrm{y} \in \mathrm{H},(1-\mathrm{rs})(\mathrm{y})=\mathrm{y}-\mathrm{r}(\mathrm{s}(\mathrm{y}))=\mathrm{y}-\mathrm{r}(0)=\mathrm{y} \in \mathrm{H}$ and for $\mathrm{z}=\mathrm{h}+\mathrm{a} \in \mathrm{H}+\mathrm{a}$, $(1-\mathrm{rs})(\mathrm{z})=\mathrm{z}-\mathrm{r}(\mathrm{s}(\mathrm{z}))=\mathrm{z}-\mathrm{r}(\mathrm{b})=(\mathrm{h}+\mathrm{a})-\left(\mathrm{h}^{\prime}+\mathrm{a}\right)=\mathrm{h}-\mathrm{h}^{\prime} \in \mathrm{H}$. Therefore, 1 - $r s \in(H: G)=K$ and hence $R / K$ is a right $R$-group of type-2. Since $R$ is simple, $\{0\}$ is the largest ideal of $\mathrm{R}$ contained in $(0: \mathrm{R} / \mathrm{K})=(\mathrm{K}: \mathrm{R})=\{\mathrm{t} \in \mathrm{R} \mid \mathrm{Rt} \subseteq \mathrm{K}\}$. Let $\mathrm{u}, \mathrm{v} \in \mathrm{R}$ and $(\mathrm{t}+\mathrm{K}) \mathrm{u}=(\mathrm{t}+\mathrm{K}) \mathrm{v}$ for all $\mathrm{t}+\mathrm{K} \in \mathrm{R} / \mathrm{K}$. Now $\mathrm{tu}-\mathrm{tv} \in \mathrm{K}$, for all $\mathrm{t} \in \mathrm{R}$. Suppose that $\mathrm{g} \in \mathrm{G}$ and $\mathrm{u}(\mathrm{g}) \neq \mathrm{v}(\mathrm{g})$. We can choose a $\mathrm{t} \in \mathrm{R}$ such that $(\mathrm{tu})(\mathrm{g})-(\mathrm{tv})(\mathrm{g}) \in \mathrm{H}+\mathrm{a}$, a contradiction to the fact that $\mathrm{tu}-\mathrm{tv} \in \mathrm{K}$. Therefore, $\mathrm{u}$ $=\mathrm{v}$ and hence $\mathrm{R} / \mathrm{K}$ is a right $\mathrm{R}$-group of type-2(e). Since $\mathrm{R}$ is simple, it is a right $2(\mathrm{e})$-primitive near-ring.

Example 3.2. Let $G$ be the non-abelian group of order 6. Let $N$ be the subgroup of $G$ of order 3. By Proposition 3.1, $M_{0}(G) /(N: G)$ is a right $M_{0}(G)$-group of type$2(\mathrm{e})$ and $M_{0}(G)$ is a right 2(e)-primitive near-ring. Since $N$ is the maximal (normal) subgroup of $G,(N: G)$ is the only proper (maximal) right ideal of $M_{0}(G)$. So a right $M_{0}(G)$-group of type-0 is $M_{0}(G)$-isomorphic to $M_{0}(G) /(N: G)$. Therefore, if $f+(N: G)$ is a generator of the right $M_{0}(G)$-group $M_{0}(G) /(N: G)$, then $(0: f+(N: G))=(N: G) \neq\{0\}$. Note that as $M_{0}(G)$ is a simple near-ring, $\{0\}$ is the largest ideal of $M_{0}(G)$ contained in $\left(0: M_{0}(G) /(N: G)\right)$. Hence $M_{0}(G) /(N: G)$ is not a right $M_{0}(G)$-group of type- $g_{\nu}, \nu \in\{0,1,2\}$.

Now we present another example to show that there are right $R$-groups of type$\nu(e)$ which are not of type- $g_{\nu}$. The following example was considered in [3] and [11].

Example 3.3. Consider $G:=Z_{8}$, the group of integers under addition modulo 8. Now $T: G \rightarrow G$ defined by $T(g)=5 g$ for all $g \in G$ is an automorphism of $G$. $T$ fixes $0,2,4,6$ and maps 1 to 5,5 to 1,3 to 7 and 7 to 3 . Now $A:=\{I, T\}$ is an automorphism group of $G$ and $\{0\},\{2\},\{4\},\{6\},\{1,5\}$ and $\{3,7\}$ are the orbits. Let $R$ be the centralizer near-ring $M_{A}(G)$, the near-ring of all self maps of $G$ which fix 0 and commute with $T$. An element of $R$ is completely determined by its action on $\{1,2,3,4,6\}$. Note that for $f \in R$ we have $f(2), f(4), f(6)$ are arbitrary in $2 G$ and $f(1), f(3)$ are arbitrary in $G$. In [3] shown that $I:=(0: 2 G)=\{f \in R \mid f(h)=0$, for all $h \in 2 G\}$ is the only non-trivial ideal of $R$. Let $K:=(2 G: G)=\{t \in R \mid$ $t(G) \subseteq 2 G\} \neq R$. Let $t_{0}$ be the identity element in $R$. Now $t_{0}+K$ is a generator of the right $R$-group $R / K$. Let $h \in R-K$. We show now that $(h+K) R=R / K$. Since $h \notin K$, there is an $a \in G-2 G$ such that $b:=h(a) \notin 2 G$. We construct an element $s \in R$ such that $s(1)=s(3)=a$, so that $s(5)=s(7)=a+4$, and $s=0$ on $2 G$. Since s maps $G-2 G$ to $G-2 G$, we get that $t_{0}-h s \in K$ and hence $(h+K) s=t_{0}+K$. So $(h+K) R=R / K$. Therefore, $R / K$ is a right $\mathrm{R}$-group of type- $\nu$. Moreover, $(R / K) I \neq\{K\}$. Therefore, $\{0\}$ is the largest ideal of $R$ contained in $(K: R)$ and hence $J_{\nu}^{r}(R)=\{0\}$. Consider $s_{1}, s_{1} \in R$, where $s_{1}(1)=1$ and 0 on $G-\{1,5\}$ and 
$s_{2}(1)=5$ and 0 on $G-\{1,5\}$. Clearly $(h+K) s_{1}=(h+K) s_{2}$ for all $h \in R$ as $h(1)-h(5) \in 2 G$ for all $h \in R$. But $s_{1}-s_{2} \notin\{0\}$. Therefore, $R / K$ is not a right $R$-group of type- $\nu(e)$.

Proposition 3.4. Let $\mathrm{R}$ be the near-ring considered in the Example 3.3 and let $\mathrm{K}$ be a right ideal of $\mathrm{R}$. Then $\mathrm{H}_{1}:=\{\mathrm{f}(\mathrm{g}) \mid \mathrm{f} \in \mathrm{K}, \mathrm{g} \in \mathrm{G}\} \subseteq \mathrm{G}$ and $\mathrm{H}_{2}:=\{\mathrm{f}(\mathrm{g}) \mid \mathrm{f}$ $\in \mathrm{K}, \mathrm{g} \in 2 \mathrm{G}\} \subseteq 2 \mathrm{G}$ are (normal) subgroups of $\mathrm{G}$ and $2 \mathrm{G}$ respectively.

Proof. We show that $\mathrm{H}_{1}$ is a subgroup of $\mathrm{G}$. Since $0 \in \mathrm{H}_{1}, \mathrm{H}_{1}$ is non-empty. Let $\mathrm{h}_{1}, \mathrm{~h}_{2} \in \mathrm{H}_{1}$. We get $\mathrm{f}_{1}, \mathrm{f}_{2} \in \mathrm{K}$ and $\mathrm{g}_{1}, \mathrm{~g}_{2} \in \mathrm{G}$ such that $\mathrm{h}_{1}=\mathrm{f}_{1}\left(\mathrm{~g}_{1}\right)$ and $\mathrm{h}_{2}=$ $\mathrm{f}_{2}\left(\mathrm{~g}_{2}\right)$. Clearly, $-\mathrm{h}_{1}=\left(-\mathrm{f}_{1}\right)\left(\mathrm{g}_{1}\right) \in \mathrm{H}_{1}$ as $-\mathrm{f}_{1} \in \mathrm{K}$. Suppose that one of the $\mathrm{g}_{i}$ is in $\mathrm{G}$ $2 \mathrm{G}$. With out loss of generality, suppose that $\mathrm{g}_{1} \in \mathrm{G}-2 \mathrm{G}$. We get $\mathrm{f}_{3} \in \mathrm{R}$ such that $\mathrm{f}_{3}\left(\mathrm{~g}_{1}\right)=\mathrm{g}_{2}$. Now $\mathrm{f}_{1}-\mathrm{f}_{2} \mathrm{f}_{3} \in \mathrm{K}$ and $\mathrm{h}_{1}-\mathrm{h}_{2}=\left(\mathrm{f}_{1}-\mathrm{f}_{2} \mathrm{f}_{3}\right)\left(\mathrm{g}_{1}\right) \in \mathrm{H}_{1}$. Assume now that $\mathrm{g}_{1}, \mathrm{~g}_{2} \in 2 \mathrm{G}$. So, $\mathrm{h}_{1}, \mathrm{~h}_{2} \in 2 \mathrm{G}$. If $\mathrm{g}_{1}=0$, then $\mathrm{h}_{1}-\mathrm{h}_{2}=-\mathrm{h}_{2} \in \mathrm{H}_{1}$. Suppose that $\mathrm{g}_{1}$ $\neq 0$. So, we get $\mathrm{f}_{4} \in \mathrm{R}$ such that $\mathrm{f}_{4}\left(\mathrm{~g}_{1}\right)=\mathrm{g}_{2}$. Now $\mathrm{f}_{1}-\mathrm{f}_{2} \mathrm{f}_{4} \in \mathrm{K}$ and $\mathrm{h}_{1}-\mathrm{h}_{2}=\left(\mathrm{f}_{1}\right.$ - $\left.\mathrm{f}_{2} \mathrm{f}_{4}\right)\left(\mathrm{g}_{1}\right) \in \mathrm{H}_{1}$. Therefore, $\mathrm{H}_{1}$ is a subgroup of G. Similarly, we get that $\mathrm{H}_{2}$ is a subgroup of $2 \mathrm{G}$.

Proposition 3.5. Let R, $\mathrm{K}, \mathrm{H}_{1}$ and $\mathrm{H}_{2}$ be as defined in Proposition 3.4. If $\mathrm{H}_{1}=$ $\mathrm{G}$ and $\mathrm{H}_{2}=2 \mathrm{G}$, then $\mathrm{K}=\mathrm{R}$.

Proof. Suppose that $\mathrm{H}_{1}=\mathrm{G}$ and $\mathrm{H}_{2}=2 \mathrm{G}$. We have $1,3 \in \mathrm{H}_{1}$. So, for $\mathrm{i} \in\{1,3\}$, we get $\mathrm{f}_{i} \in \mathrm{K}$ such that $\mathrm{f}_{i}\left(\mathrm{~g}_{i}\right)=\mathrm{i}$, where $\mathrm{g}_{i} \in\{1,3,5,7\}=\mathrm{G}-2 \mathrm{G}$. For $\mathrm{i}=1,3$ we also get $\mathrm{m}_{i} \in \mathrm{R}$ such that $\mathrm{m}_{i}(\mathrm{i})=\mathrm{g}_{i}$, so that $\mathrm{m}_{i}(\mathrm{i}+4)=\mathrm{g}_{i}+4$ and $\mathrm{m}_{i}=0$ on $\mathrm{G}$ $-\{\mathrm{i}, \mathrm{i}+4\}$. Now $\mathrm{f}_{i} \mathrm{~m}_{i} \in \mathrm{K}, \mathrm{i}=1,3$. Clearly, $\mathrm{f}_{1} \mathrm{~m}_{1}+\mathrm{f}_{3} \mathrm{~m}_{3}$ fixes all the elements of $\mathrm{G}-2 \mathrm{G}$ and maps all the elements of $2 \mathrm{G}$ to 0 . We have $2,4,6 \in \mathrm{H}_{2}=2 \mathrm{G}=\{0,2$, $4,6\}$. For $\mathrm{i}=2,4,6$ we get $\mathrm{f}_{i} \in \mathrm{K}$ such that $\mathrm{f}_{i}\left(\mathrm{~g}_{i}\right)=\mathrm{i}, \mathrm{g}_{i} \in 2 \mathrm{G}$. So, for $\mathrm{i}=2,4$, 6 we get $\mathrm{m}_{i} \in \mathrm{R}$ such that $\mathrm{m}_{i}(\mathrm{i})=\mathrm{g}_{i}$ and $\mathrm{m}_{i}$ is 0 on $\mathrm{G}-\{\mathrm{i}\}$. Now $\mathrm{f}_{i} \mathrm{~m}_{i} \in \mathrm{K}, \mathrm{i}=$ $2,4,6 . \mathrm{f}_{2} \mathrm{~m}_{2}+\mathrm{f}_{4} \mathrm{~m}_{4}+\mathrm{f}_{6} \mathrm{~m}_{6}$ fixes all the elements of $2 \mathrm{G}$ and maps all the elements of $G-2 G$ to 0 . Therefore, the identity map I of $G$ can be expressed as $I=f_{1} m_{1}+$ $\mathrm{f}_{2} \mathrm{~m}_{2}+\mathrm{f}_{3} \mathrm{~m}_{3}+\mathrm{f}_{4} \mathrm{~m}_{4}+\mathrm{f}_{6} \mathrm{~m}_{6} \in \mathrm{K}$. Hence, $\mathrm{K}=\mathrm{R}$.

Proposition 3.6. Let $\mathrm{R}, \mathrm{K}, \mathrm{H}_{1}$ and $\mathrm{H}_{2}$ be as defined in Proposition 3.4. If $\mathrm{K}$ is a maximal right ideal of $\mathrm{R}$, then $\mathrm{K}=(2 \mathrm{G}: \mathrm{G})=\{\mathrm{f} \in \mathrm{R} \mid \mathrm{f}(\mathrm{G}) \subseteq 2 \mathrm{G}\}$ or $(4 \mathrm{G}: 2 \mathrm{G})$ $=\{\mathrm{f} \in \mathrm{R} \mid \mathrm{f}(2 \mathrm{G}) \subseteq 4 \mathrm{G}\}$

Proof. Suppose that $\mathrm{K}$ is a maximal right ideal of $\mathrm{R}$. Clearly, if $\mathrm{H}$ and $\mathrm{T}$ are (normal) subgroups of $\mathrm{G}$ and $2 \mathrm{G}$ respectively, then $(\mathrm{H}: \mathrm{G})=\{\mathrm{f} \in \mathrm{R} \mid \mathrm{f}(\mathrm{G}) \subseteq \mathrm{H}\}$ and $(\mathrm{T}$ : $2 \mathrm{G})=\{\mathrm{f} \in \mathrm{R} \mid \mathrm{f}(2 \mathrm{G}) \subseteq \mathrm{T}\}$ are right ideals of $\mathrm{R}$. Now $2 \mathrm{G}$ and $4 \mathrm{G}$ are the maximal (normal) subgroups of $\mathrm{G}$ and $2 \mathrm{G}$ respectively. We have $\mathrm{K} \subseteq\left(\mathrm{H}_{1}: \mathrm{G}\right)$ and $\mathrm{K} \subseteq\left(\mathrm{H}_{2}\right.$ : $2 \mathrm{G}$ ). Since $\mathrm{K}$ is a maximal right ideal of $\mathrm{R}$, by Proposition 3.5, either $\mathrm{H}_{1} \neq \mathrm{G}$ or $\mathrm{H}_{2} \neq 2 \mathrm{G}$.

Case(i) Suppose that $\mathrm{H}_{2} \neq 2 \mathrm{G}$. Since $\mathrm{K}$ is a maximal right ideal of $\mathrm{R}$ and $\mathrm{K} \subseteq\left(\mathrm{H}_{2}\right.$ $: 2 \mathrm{G}) \neq \mathrm{R}$, we get that $\mathrm{H}_{2}=4 \mathrm{G}$ and $\mathrm{K}=(4 \mathrm{G}: 2 \mathrm{G})$.

case(ii) Suppose that $H_{1} \neq G$. Since $K$ is a maximal right ideal of $R$ and $K \subseteq\left(H_{1}\right.$ $: G) \neq R$, we get that $\mathrm{H}_{1}=2 \mathrm{G}$ and $\mathrm{K}=(2 \mathrm{G}: \mathrm{G})$.

Therefore, either $\mathrm{K}=(2 \mathrm{G}: \mathrm{G})$ or $(4 \mathrm{G}: 2 \mathrm{G})$. 
Proposition 3.7. Let $\mathrm{R}$ be the near-ring considered in the Example 3.3. Let $\mathrm{U}$ $=(4 \mathrm{G}: 2 \mathrm{G})=\{\mathrm{f} \in \mathrm{R} \mid \mathrm{f}(2 \mathrm{G}) \subseteq 4 \mathrm{G}\}$. Then $\mathrm{U}$ is a maximal right ideal of $\mathrm{R}$ and $\mathrm{R} / \mathrm{U}$ is a right $\mathrm{R}$-group of type- $2(\mathrm{e})$.

Proof. Clearly, $\mathrm{U}$ is a right ideal of $\mathrm{R}$. Consider the right R-group $\mathrm{R} / \mathrm{U}$. We prove that $\mathrm{R} / \mathrm{U}$ is a right $\mathrm{R}$-group of type-2. Since $\mathrm{R}$ has identity $\mathrm{I}, \mathrm{I}+\mathrm{U}$ is a generator of the right $\mathrm{R}$-group $\mathrm{R} / \mathrm{U}$ and hence $\mathrm{R} / \mathrm{U}$ is a monogenic right $\mathrm{R}$-group. Let $0 \neq$ $\mathrm{f}+\mathrm{U} \in \mathrm{R} / \mathrm{U}$. So, $\mathrm{f} \notin \mathrm{U}$. We get $0 \neq \mathrm{a} \in 2 \mathrm{G}$ such that $\mathrm{b}:=\mathrm{f}(\mathrm{a}) \notin 4 \mathrm{G}$. So, $2 \mathrm{G}=$ $\{0, \mathrm{~b}, 2 \mathrm{~b}, 3 \mathrm{~b}\}$ as 2 and 6 are generators of $2 \mathrm{G}$. Construct $\mathrm{r} \in \mathrm{R}$ by $\mathrm{r}(\mathrm{b})=\mathrm{a}, \mathrm{r}(2 \mathrm{~b})$ $=0, r(3 b)=a$ and $\mathrm{r}=0$ on $\mathrm{G}-\{0,1,3,5,7\}$. Now $(\mathrm{I}-\mathrm{fr})(\mathrm{x}) \in 4 \mathrm{G}$ for all $\mathrm{x} \in$ $2 \mathrm{G}$. Therefore, $\mathrm{I}-\mathrm{fr} \in \mathrm{U}$ and hence $(\mathrm{f}+\mathrm{U}) \mathrm{r}=\mathrm{I}+\mathrm{U}$. This shows that $(\mathrm{f}+\mathrm{U}) \mathrm{R}$ $=\mathrm{R} / \mathrm{U}$. So, $\mathrm{R} / \mathrm{U}$ is a right $\mathrm{R}$-group of type- 2 . We know that $\mathrm{P}:=(0: 2 \mathrm{G})$ is the only non-trivial ideal of $\mathrm{R}$. Therefore, $\mathrm{P}$ is the largest ideal of $\mathrm{R}$ contained in $\mathrm{U}=$ $(4 \mathrm{G}: 2 \mathrm{G})$ and hence $\mathrm{P}$ is the largest ideal of $\mathrm{R}$ contained in $(0: \mathrm{R} / \mathrm{U})=(\mathrm{U}: \mathrm{R})$ $=\{\mathrm{f} \in \mathrm{R} \mid \mathrm{Rf} \subseteq \mathrm{U}\}$. Let $0 \neq \mathrm{s}+\mathrm{U} \in \mathrm{R} / \mathrm{U}$ and $\mathrm{f}, \mathrm{h} \in \mathrm{R}$. Suppose that $(\mathrm{s}+\mathrm{U}) \mathrm{rf}$ $=(\mathrm{s}+\mathrm{U}) \mathrm{rh}$ for all $\mathrm{r} \in \mathrm{R}$. So, srf $-\mathrm{srh} \in \mathrm{U}$ for all $\mathrm{r} \in \mathrm{R}$. We show that $\mathrm{f}-\mathrm{h} \in \mathrm{P}$. If possible, suppose that $\mathrm{f}-\mathrm{h} \notin \mathrm{P}$. We get $0 \neq \mathrm{a} \in 2 \mathrm{G}$ such that $(\mathrm{f}-\mathrm{h})(\mathrm{a})=\mathrm{f}(\mathrm{a})$ $\mathrm{h}(\mathrm{a}) \neq 0$ with $\mathrm{h}(\mathrm{a}) \neq 0$. Let $\mathrm{s}(\mathrm{c}) \notin\{0,4\}$ for some $\mathrm{c} \in 2 \mathrm{G}$. Choose $\mathrm{r} \in \mathrm{R}$ such that $\mathrm{r}(\mathrm{f}(\mathrm{a}))=0$ and $\mathrm{r}(\mathrm{h}(\mathrm{a}))=\mathrm{c}$. Now $(\operatorname{srf})(\mathrm{a})=0$ and $(\operatorname{srh})(\mathrm{a})=\mathrm{s}(\mathrm{c})$. So, $(\operatorname{srf}-\operatorname{srh})(\mathrm{a})$ $=0-\mathrm{s}(\mathrm{c}) \notin\{0,4\}$, a contradiction to the fact that srf - $\mathrm{srh} \in \mathrm{U}$. Therefore, $\mathrm{f}(\mathrm{a})=$ $\mathrm{h}(\mathrm{a})$ for all $\mathrm{a} \in 2 \mathrm{G}$. Hence $\mathrm{f}-\mathrm{h} \in \mathrm{P}$. So, $\mathrm{R} / \mathrm{U}$ is a right R-group of type-2(e).

Proposition 3.8. Let $\mathrm{R}$ be the near-ring considered in Example 3.3. Then $\mathrm{J}_{\nu}^{r}(\mathrm{R})$ $=\{0\}$ and $\mathrm{J}_{\nu(e)}^{r}(\mathrm{R})=(0: 2 \mathrm{G}) \neq\{0\}$.

Proof. We know that $\{0\}$ and $\mathrm{I}:=(0: 2 \mathrm{G})=\{\mathrm{f} \in \mathrm{R} \mid \mathrm{f}(2 \mathrm{G})=\{0\}\}$ are the only proper ideals of $R$. Let $\mathrm{K}_{1}:=(2 \mathrm{G}: \mathrm{G})=\{\mathrm{f} \in \mathrm{R} \mid \mathrm{f}(\mathrm{G}) \subseteq 2 \mathrm{G}\}$ and $\mathrm{K}_{2}:=(4 \mathrm{G}$ : $2 \mathrm{G})=\{\mathrm{f} \in \mathrm{R} \mid \mathrm{f}(2 \mathrm{G}) \subseteq 4 \mathrm{G}\}$. By Proposition 3.6, a maximal right ideal of $\mathrm{R}$ is either $K_{1}$ or $K_{2}$. So, a right R-group of type-0 is isomorphic to $R / K_{1}$ or $R / K_{2}$. By Example $3.3, \mathrm{R} / \mathrm{K}_{1}$ is a right R-group of type- 2 but not of type- $2(\mathrm{e})$. Since $\{0\}$ is the largest ideal of $\mathrm{R}$ contained in $\mathrm{K}_{1},\{0\}$ is a right 2-primitive ideal of $\mathrm{R}$ but not a right 2(e)-primitive ideal of $\mathrm{R}$. By Proposition $3.7, \mathrm{R} / \mathrm{K}_{2}$ is a right $\mathrm{R}$-group of type-2(e). Since $\mathrm{I}=(0: 2 \mathrm{G})$ is the largest ideal of $\mathrm{R}$ contained in $\mathrm{K}_{2}, \mathrm{I}$ is a right $2(\mathrm{e})$-primitive ideal of R. Therefore, $J_{\nu}^{r}(\mathrm{R})=\{0\}$ and $J_{\nu(e)}^{r}(R)=(0: 2 G)$.

Proposition 3.9. Let $\mathrm{R}$ be the near-ring considered in Example 3.3. Then $J_{g_{\nu}}^{r}(R)=R, \nu \in\{0,1,2\}$.

Proof. Let $R$ be the near-ring considered in the Example 3.3 and $K=(2 G: G)$, $U=(4 G: 2 G)$. As seen above $K, U$ are the only maximal right ideals of $R$ and $R / K$ is a right $R$-group of type- 2 but not of type-2(e), where as $R / U$ is a right $R$-group of type-2(e). If $f+K$ is a generator of the right $R$-group $R / K$, then the maximal right ideal $(0: f+K)$ must be either $K$ or $U$. Since $0(K)=2^{10} \neq 2^{9}=0(U)$, and $R /(0: f+K)$ is right $R$-isomorphic $R / K,(0: f+K)=K$. Hence $R / K$ is not a right R-group of type- $g_{\nu}$ as $\{0\},(0: 2 G)$ and $R$ are the only ideals of $R$. By a similar argument we get that $R / U$ is not a right $R$-group of type- $g_{\nu}$. So $J_{g_{\nu}}^{r}(R)=R$. 
4. $J_{g_{\nu}}^{r}$-semisimple Near-rings, $\nu \in\{0,1,2\}$

In this section we present structure theorems for $J_{g_{\nu}}^{r}$-semisimple near-rings.

Proposition 4.1. Let $R(\neq\{0\})$ be a $J_{g_{\nu}}^{r}$-semisimple near-rings satisfying DCC on right ideals of $R, \nu \in\{0,1,2\}$. Then $R$ is a finite direct sum of minimal right ideals which are right $R$-groups of type- $g_{\nu}$.

Proof. Let $P_{i}, i \in I$ be the collection of right $g_{\nu}$-primitive ideals of $R$. Since $R$ is a $J_{g_{\nu}}^{r}$-semisimple near-ring, $\cap\left\{P_{i} \mid i \in I\right\}=\{0\}$. We get a right $R$-group $G_{i}$ of type- $g_{\nu}$ such that $P_{i}=\left(0: G_{i}\right):=\left\{r \in R \mid G_{i} r=\{0\}\right\}, i \in I$. Let $A_{i}$ be the set of generators of $G_{i}, i \in I$. Now $P_{i}=\left(0: A_{i}\right):=\left\{r \in R \mid A_{i} r=\{0\}\right\}$. Note that for each $a \in A_{i},(0: a):=\{r \in R \mid a r=0\}$ is a right $g_{\nu}$-modular right ideal of $R$ and the right $R$-group $R /(0: a)$ is right $R$-isomorphic to $G_{i}, i \in I$. Since each $P_{i}$ is an intersection of right $g_{\nu}$-modular right ideal of $R$ and $\cap\left\{P_{i} \mid i \in I\right\}=\{0\}$, the intersection of all right $g_{\nu}$-modular right ideal of $R$ is zero. We get a finite number of right $g_{\nu^{-}}$ modular right ideals $K_{1}, K_{2}, \ldots, K_{n}$ of $R$ such that $\cap\left\{K_{j} \mid j=1,2, \ldots, n\right\}=\{0\}$. Let $T_{i}:=K_{1} \cap K_{2} \cap \ldots \cap K_{i-1} \cap K_{i+1} \cap \ldots \cap K_{n}, i=1,2, \ldots, n$. We may assume that $T_{i} \neq\{0\}$ for all $i=1,2, \ldots, n$. Now by Proposition 3.12[(2)] of [8], $R=T_{1} \oplus T_{2} \oplus \ldots \oplus T_{n}$, a direct sum of minimal right ideals $T_{i}$ of $R$ which are right $R$-groups of type- $g_{\nu}$.

In [8](Definition 3.5), if $R$ is a direct sum of $n$ minimal right ideals of $R$, then the dimension of $R$ is defined as $n$ and is denoted by $\operatorname{dim} R$.

Definition 4.2. A distributive idempotent $e$ of $R$ is called right $g_{\nu}$-primitive if $e R$ is a right $R$-group of type- $g_{\nu}, \nu \in\{0,1,2\}$.

Theorem 4.3. Let $R$ be a right $g_{\nu}$-primitive near-rings satisfying $D C C$ on right ideals of $R, \nu \in\{0,1,2\}$. Then $R$ is a simple near-ring with identity and $R$ has a subnear-ring which is isomorphic to the matrix near-ring $M_{n}(S)$, where $S=e R e, e$ is a right $g_{\nu}$-primitive idempotent and $n=\operatorname{dim} R$. If, in addition, $R$ is distributively generated, then $R$ isomorphic to $M_{n}(S)$.

Proof. $R$ satisfies the hypothesis of Theorem 4.3 of [8] and hence the conclusion follows from it.

Theorem 4.4. Let $R$ be a finite right $g_{2}$-primitive near-ring and eRe be a nonring. Then $R$ is (isomorphic to) the matrix near-ring $M_{n}(F)$, where $n=\operatorname{dim} R$, $F:=e$ Re is a near-field and $e$ is a right $g_{2}$-primitive idempotent in $R$.

Proof. Proof follows from Theorem 4.16 of [8].

Theorem 4.5. Let $R(\neq\{0\})$ be a $J_{g_{\nu}}^{r}$-semisimple near-rings satisfying DCC on right ideals of $R, \nu \in\{0,1,2\}$. Then $R$ is a direct sum of minimal ideals which are simple right $g_{\nu}$-primitive near-rings with identity. 
Proof. Let $P_{i}, i \in I$ be the collection of right $g_{\nu}$-primitive ideals of $R, \nu \in\{0,1,2\}$. Now $\cap\left\{P_{i} \mid i \in I\right\}=\{0\}$. Since $R$ has DCC on right ideals of $R$, we get a finite number of right $g_{\nu}$-primitive ideals of $P_{1}, P_{2}, \ldots, P_{n}$ of $R$ such that $P_{1} \cap P_{2} \cap \ldots \cap P_{n}=$ $\{0\}$. We may assume that $K_{j}:=P_{1} \cap P_{2} \cap \ldots \cap P_{j-1} \cap P_{j+1} \cap \ldots \cap P_{n} \neq\{0\}, j=$ $1,2, \ldots, n$. By Theorem $4.3, R / P_{i}$ is a simple near-ring with identity as $R / P_{i}$ is a right $g_{\nu}$-primitive near-ring with DCC on right ideals. Now by Theorem 2.50 of Pilz [4], $R=K_{1} \oplus K_{2} \oplus \ldots \oplus K_{n}, K_{i}$ are minimal ideals of $R$ and are simple right $g_{\nu}$-primitive near-rings with identity.

Acknowledgments. The first author would like to thank the Management of the Nagarjuna Education Society, Guntur, for providing necessary facilities. The first author acknowledge the U.G.C., New Delhi, for the Major Research Project Grant No. F. 39-51/2010 (SR), 24 December, 2010.

\section{References}

[1] G. L. Booth and N. J. Groenewald, Special radicals of near-rings, Math. Japonica, 37(4)(1992), 701-706.

[2] G. L. Booth, N. J. Groenewald, and S. Veldsman, A Kurosh-Amitsur prime radical for near-rings, Comm. Algebra, 18(9)(1990), 3111-3122.

[3] K. Kaarli, On Jacobson-type radicals of near-rings, Acta Math. Hungar., 50(1987), $71-78$.

[4] G. Pilz, Near-rings, revised ed., North-Holland, Amsterdam, 1983.

[5] R. Srinivasa Rao and K. Siva Prasad, Hereditary right Jacobson radicals of type-1(e) and $2(e)$ for right near-rings, submitted for publication.

[6] R. Srinivasa Rao and K. Siva Prasad, A radical for right near-rings: The right Jacobson radical of type-0, Int. J. Math. Math. Sci., 2006(16)(2006), 1-13, Article ID 68595.

[7] R. Srinivasa Rao and K. Siva Prasad, Two more radicals for right near-rings: The right Jacobson radicals of type-1 and 2, Kyungpook Math. J., 46(4)(2006), 603-613.

[8] R. Srinivasa Rao and K. Siva Prasad, Right semisimple right near-rings, Southeast Asian Bull. Math., 33(6)(2009), 1189-1205.

[9] R. Srinivasa Rao, K. Siva Prasad, and T. Sinivas, Kurosh-Amitsur right Jacobson radical of type-0 for right near-rings, Int. J. Math. Math. Sci., 2008(1)(2008), 1-6, Article ID 741609.

[10] R. Srinivasa Rao, K. Siva Prasad, and T. Sinivas, Kurosh-Amitsur right Jacobson radicals of type-1 and 2 for right near-rings, Result. Math., 51(3-4)(2008), 309-317.

[11] R. Srinivasa Rao, K. Siva Prasad, and T. Sinivas, Hereditary right Jacobson radical of type-O(e) for right near-rings, Beitr. Algebra Geom., 50(1)(2009), 11-23.

[12] S. Veldsman, Modulo-constant ideal-hereditary radicals of near-rings, Quaest. Math., 11(3)(1988), 253-278. 